\title{
A natural series for the natural logarithm
}

\author{
Oliver T. Dasbach \\ kasten@math.lsu.edu \\ Louisiana State University, Department of Mathematics \\ Baton Rouge, LA 70803, http://www.math.lsu.edu/ kasten \\ Submitted: Feb 22, 2007; Accepted: Feb 27, 2008; Published: Mar 7, 2008 \\ Mathematics Subject Classification: 57M25, 57M50, 40A05, 05A10
}

\begin{abstract}
Rodriguez Villegas expressed the Mahler measure of a polynomial in terms of an infinite series. Lück's combinatorial $L^{2}$-torsion leads to similar series expressions for the Gromov norm of a knot complement.

In this note we show that those formulae yield interesting power series expansions for the logarithm function. This generalizes an infinite series of Lehmer for the natural logarithm of 4 .
\end{abstract}

\section{The abelian case: the Mahler measure}

For a Laurent polynomial $P$ in the group ring $\mathbb{C}\left[\mathbb{Z}^{r}\right]$ let the conjugate $P^{*}$ be defined by sending every $g \in \mathbb{Z}^{r}$ to $g^{-1}$ and every coefficient $a_{g}$ to its complex conjugate $\overline{a_{g}}$.

The (logarithmic) Mahler measure (see e.g. [EW99]) of $P$ is given by:

$$
m(P)=\int_{0}^{1} \cdots \int_{0}^{1} \ln \left|P\left(e^{2 \pi i t_{1}}, \ldots, e^{2 \pi i t_{r}}\right)\right| d t_{1} \cdots d t_{r} .
$$

The following theorem is due to Rodriguez Villegas [RV99]. Independently, it also appears in the study of the combinatorial $L^{2}$-torsion due to Lück. Further discussions are given in [DL08]. We include a proof along the lines of [RV99] for completeness.

Theorem 1.1 ([RV99], see also [Lüc02, Den06]). For $k$ greater than the $l^{1}$-norm of the coefficients of $P$ we have

$$
2 m(P)=m\left(P P^{*}\right)=2 \ln (k)-\sum_{n=1}^{\infty} \frac{1}{n}\left[\left(1-\frac{1}{k^{2}} P P^{*}\right)^{n}\right]_{0},
$$

where $[P]_{0}$ denotes the constant coefficient of the Laurent polynomial P. 
Proof. Since

$$
P P^{*}=k^{2}\left(1-\left(1-\frac{1}{k^{2}} P P^{*}\right)\right)
$$

we have

$$
m\left(P P^{*}\right)=2 \ln (k)+m\left(1-\left(1-\frac{1}{k^{2}} P P^{*}\right)\right) .
$$

Set $Q:=1-\frac{1}{k^{2}} P P^{*}$ and let

$$
\begin{aligned}
u(Q, x) & :=\int_{0}^{1} \cdots \int_{0}^{1} \frac{1}{1-x Q\left(e^{2 \pi i t_{1}}, \ldots, e^{2 \pi i t_{r}}\right)} d t_{1} \cdots d t_{r} \\
& =\sum_{n=0}^{\infty} x^{n} \int_{0}^{1} \cdots \int_{0}^{1} Q\left(e^{2 \pi i t_{1}}, \ldots, e^{2 \pi i t_{r}}\right)^{n} d t_{1} \cdots d t_{r} \\
& =\sum_{n=0}^{\infty} x^{n}\left[Q^{n}\right]_{0}
\end{aligned}
$$

The choice of $k$ ensures convergence.

Now

$$
\begin{aligned}
m(1-x Q) & =\int_{0}^{1} \cdots \int_{0}^{1} \ln \left|1-Q\left(e^{2 \pi i t_{1}}, \ldots, e^{2 \pi i t_{r}}\right)\right| d t_{1} \cdots d t_{r} \\
& =\int_{0}^{1} \cdots \int_{0}^{1} \ln \left(1-Q\left(e^{2 \pi i t_{1}}, \ldots, e^{2 \pi i t_{r}}\right)\right) d t_{1} \cdots d t_{r} \\
& =-\int_{0}^{x}(u(Q, z)-1) \frac{d z}{z} \\
& =-\sum_{n=1}^{\infty} \frac{1}{n} x^{n}\left[Q^{n}\right]_{0}
\end{aligned}
$$

setting $x:=1$ yields the result.

\section{A power series for the natural logarithm}

Here we study an application of Equation (1) which leads to an interesting identity. For the polynomial $1+a$ the right-hand side of Equation (1) yields:

$$
\begin{aligned}
& 2 \ln (k)-\sum_{n \geq 1} \frac{1}{n} \operatorname{tr}_{\mathbb{C} G}\left(1-\frac{1}{k^{2}}(1+a)\left(1+a^{-1}\right)\right)^{n} \\
= & 2 \ln (k)-\sum_{n \geq 1} \frac{1}{n} \operatorname{tr}_{\mathbb{C} G}\left(\sum_{j=0}^{n}\left(\begin{array}{c}
n \\
j
\end{array}\right)\left(-\frac{1}{k^{2}}\right)^{j}\left(2+a+a^{-1}\right)^{j}\right)
\end{aligned}
$$


Hence, with a formal $\sqrt{a}$ :

$$
\begin{aligned}
& =2 \ln (k)-\sum_{n \geq 1} \frac{1}{n} \operatorname{tr}_{\mathbb{C} G}\left(\sum_{j=0}^{n}\left(\begin{array}{c}
n \\
j
\end{array}\right)\left(-\frac{1}{k^{2}}\right)^{j}\left(\sqrt{a}+\frac{1}{\sqrt{a}}\right)^{2 j}\right) \\
& =2 \ln (k)-\sum_{n \geq 1} \frac{1}{n} \sum_{j=0}^{n}\left(\begin{array}{c}
n \\
j
\end{array}\right)\left(-\frac{1}{k^{2}}\right)^{j}\left(\begin{array}{c}
2 j \\
j
\end{array}\right)
\end{aligned}
$$

On the other hand we know by Theorem 1.1 that the right-hand side of Equation (1) equals the logarithm of the Mahler measure of $(1+a)$ which is 0 . This shows:

Theorem 2.1. For $x \geq 4$ a power series for the logarithm is given by:

$$
\ln x=\sum_{n \geq 1} \sum_{j=0}^{n} \frac{1}{n}\left(\begin{array}{c}
n \\
j
\end{array}\right)\left(\begin{array}{c}
2 j \\
j
\end{array}\right)\left(-\frac{1}{x}\right)^{j}
$$

The case $x=4$ is somewhat special. Equation (2) at $x=k^{2}=4$ equals:

$$
\begin{aligned}
& 2 \ln 2-\sum_{n \geq 1} \frac{1}{n} \operatorname{tr}_{\mathbb{C} G}\left(\frac{a-2+a^{-1}}{-4}\right)^{n} \\
= & 2 \ln 2-\sum_{n \geq 1} \frac{1}{n} \operatorname{tr}_{\mathbb{C} G}\left((-1)^{n} \frac{(\sqrt{a}-1 / \sqrt{a})^{2 n}}{4^{n}}\right) \\
= & 2 \ln 2-\sum_{n \geq 1} \frac{1}{n}\left(\begin{array}{c}
2 n \\
n
\end{array}\right) \frac{1}{4^{n}}
\end{aligned}
$$

Thus, we recover an identity which is well-known to Mathematica [Wol99] and was derived by Lehmer in [Leh85]:

$$
\ln 4=\sum_{n=1}^{\infty} \frac{1}{n}\left(\begin{array}{c}
2 n \\
n
\end{array}\right) \frac{1}{4^{n}}
$$

The function also converges at $x=k^{2}=2$ and yields:

$$
\ln 2=\frac{1}{2} \ln 4=\sum_{n=1}^{\infty} \frac{1}{2 n}\left(\begin{array}{c}
2 n \\
n
\end{array}\right) \frac{1}{4^{n}} .
$$

\subsection{A generating function}

It is interesting to look at the series for the natural logarithm in Theorem 2.1 from a generating function point of view:

Let

$$
f_{n}(y)=\sum_{j=0}^{n} \frac{1}{n}\left(\begin{array}{c}
n \\
j
\end{array}\right)\left(\begin{array}{c}
2 j \\
j
\end{array}\right) y^{j}
$$


and

$$
F(x, y)=\sum_{n \geq 1} f_{n}(y) x^{n}
$$

its generating function.

Similar to Example 5, Section 4.3 in [Wil94] we have:

$$
\begin{aligned}
F(x, y) & =\sum_{n \geq 1} \sum_{j=1}^{n} \frac{1}{n}\left(\begin{array}{c}
n \\
j
\end{array}\right)\left(\begin{array}{c}
2 j \\
j
\end{array}\right) y^{j} x^{n}+\sum_{n \geq 1} \frac{1}{n} x^{n} \\
& =\sum_{j=1}^{\infty}\left(\begin{array}{c}
2 j \\
j
\end{array}\right) y^{j} \sum_{n=j}^{\infty} \frac{1}{n}\left(\begin{array}{c}
n \\
j
\end{array}\right) x^{n}-\ln (1-x) \\
& =\sum_{j=1}^{\infty}\left(\begin{array}{c}
2 j \\
j
\end{array}\right) y^{j} \frac{1}{j}\left(\frac{x}{1-x}\right)^{j}-\ln (1-x) \\
& =\sum_{j=1}^{\infty}\left(\begin{array}{c}
2 j \\
j
\end{array}\right) \frac{1}{j}\left(\frac{x y}{1-x}\right)^{j}-\ln (1-x) \\
& =\ln 4-2 \ln \left(1+\sqrt{1-4 \frac{x y}{1-x}}\right)-\ln (1-x) \\
& =\ln 4-2 \ln (\sqrt{1-x}+\sqrt{1-x-4 x y})
\end{aligned}
$$

Here we make use of the identity [Leh85]

$$
\sum_{j=1}^{\infty} \frac{1}{j}\left(\begin{array}{c}
2 j \\
j
\end{array}\right) z^{j}=2 \log \left(\frac{1-\sqrt{1-4 z}}{2 z}\right)
$$

\subsection{Triple Sum Series}

By working with the polynomials $1+a+a^{2}+\cdots+a^{n}$ in Equation (1) one readily obtains other infinite series for the logarithm. For example if $1+a+a^{2}$ and we see for $k \geq \sqrt{3}$ :

$$
\begin{aligned}
2 \ln k & =\sum_{n \geq 1} \frac{1}{n} \operatorname{tr}_{\mathbb{C} G}\left(1-\frac{1}{k^{2}}\left(1+a+a^{2}\right)\left(1+a^{-1}+a^{-2}\right)\right)^{n} \\
& =\sum_{n \geq 1} \frac{1}{n} \operatorname{tr}_{\mathbb{C} G}\left(\sum_{j=0}^{n}\left(\begin{array}{c}
n \\
j
\end{array}\right)\left(-\frac{1}{k^{2}}\right)^{j}\left(a^{-1}+1+a\right)^{2 j}\right) \\
& =\sum_{n \geq 1} \frac{1}{n} \sum_{j=0}^{n}\left(\begin{array}{c}
n \\
j
\end{array}\right)\left(-\frac{1}{k^{2}}\right)^{j} \sum_{l=0}^{j}\left(\begin{array}{c}
2 j \\
2 j-2 l, l, l
\end{array}\right),
\end{aligned}
$$

where the terms in the inner most sum are multinomial coefficients. 


\section{Motivations: $L^{2}$-torsion of knot complements}

To generalize the setting and to explain our original motivation we need to fix some notations. Let $K$ be a knot and

$$
G=\pi_{1}\left(S^{3}-K\right)=\left\langle x_{1}, \ldots, x_{g} \mid r_{1}, \ldots, r_{g-1}\right\rangle
$$

be a presentation of the fundamental group of the knot complement. For a square matrix $M$ with entries in $\mathbb{C} G$ the trace $\operatorname{tr}_{\mathbb{C} G}(M)$ denotes the coefficient of the unit element in the sum of the diagonal elements. The matrix $A^{*}=\left(\bar{a}_{j, i}\right)$ is the conjugate transpose of $A=\left(a_{i, j}\right)$ with conjugation

$$
\overline{\sum_{g \in G} c_{g} g}=\sum_{g \in G} \overline{c_{g}} g^{-1}
$$

Let

$$
F=\left(\begin{array}{ccc}
\frac{\partial r_{1}}{\partial x_{1}} & \cdots & \frac{\partial r_{1}}{\partial x_{g}} \\
\vdots & \ddots & \vdots \\
\frac{\partial r_{g-1}}{\partial x_{1}} & \cdots & \frac{\partial r_{g-1}}{\partial x_{g}}
\end{array}\right)
$$

be the Fox Jacobian (e.g. [BZ85]) of the presentation. We obtain a $(g-1) \times(g-1)$-matrix $A$ by deleting one of the columns of $F$.

Theorem 3.1 (Lück [Lüc02]). Suppose the deleted column in the Fox Jacobian does not correspond to a generator $x_{i}$ of $G$ that represents a trivial element in $G$. Then for a hyperbolic knot $K$ and for $k$ sufficiently large it holds:

$$
\frac{1}{3 \pi} \operatorname{Vol}\left(S^{3}-K\right)=2(g-1) \ln (k)-\sum_{n=1}^{\infty} \frac{1}{n} \operatorname{tr}_{\mathbb{C} G}\left(\left(1-k^{-2} A A^{*}\right)^{n}\right)
$$

The value of $k$ can be chosen to be the product of $(g-1)^{2}$ and the maximum of the 1-norm of the entries in $A$ (see [Lüc94]).

In case of a non-hyperbolic knot, the right-hand side of (4) is proportional to the Gromov norm of the knot complement.

In the abelian case Theorem 1.1 generalizes to:

Theorem 3.2 ([Lüc02], see also [Den06]). Let $A$ have entries in $\mathbb{C}\left[x_{1}, \ldots, x_{r}\right]$. Then the right-hand side of (4) equals:

$$
2 m(\operatorname{det}(A)),
$$

where, again, $m(p)=\int_{0}^{1} \cdots \int_{0}^{1} \ln \left|p\left(e^{2 \pi i t_{1}}, \ldots, e^{2 \pi i t_{r}}\right)\right| d t_{1} \cdots d t_{r}$ is the (logarithmic) Mahler measure of the polynomial $p\left(x_{1}, \ldots, x_{r}\right)$.

Remark 3.3. Let $K=T(p, q)$ be the $(p, q)$-torus knot. It is well-known (e.g. [BZ85]) that a presentation for the knot group of $T(p, q)$ is given by:

$$
G=\pi_{1}\left(S^{3}-T(p, q)\right)=\left\langle a, b \mid a^{p}=b^{q}\right\rangle .
$$


Hence, the Fox Jacobian for $G$ is

$$
F=\left(1+a+a^{2}+\cdots+a^{p-1},-1-b-\cdots-b^{q-1}\right)
$$

and we can chose $A$ to be the $1 \times 1$ matrix:

$$
A=\left(1+a+\cdots+a^{p-1}\right) \quad \text { and thus } \quad A^{*}=\left(1+a^{-1}+\cdots+a^{-p+1}\right) .
$$

Theorems 3.1 and 3.2 now express the fact that the Gromov norm of the torus knot complements is 0 .

\subsection{Covering spaces}

For a given knot the terms in Lücks formula (4) are by no means simple to compute. A single term depends on the chosen presentation. Furthermore, the convergence of the series is slow. An indirect approach via covering spaces of the knot complement gives more flexibility for computational simplifications. We illustrate it on the example $K$ being the trefoil knot. It is well-known that the fundamental group of its knot complement is isomorphic to the braid group $B_{3}$ on three strands:

$$
B_{3}=\left\langle\sigma_{1}, \sigma_{2} \mid \sigma_{1} \sigma_{2} \sigma_{1}=\sigma_{2} \sigma_{1} \sigma_{2}\right\rangle
$$

A direct application of Formula (4) to this presentation would lead to a somewhat messy series. However, the group $B_{3}$ has a natural homomorphic image in the symmetric group $S_{3}$ and its kernel is the pure braid group $P_{3}$ of index 6 in $B_{3}$. By an application of Newworld's lemma [DM01] $P_{3}$ is isomorphic to the direct product of its center $C_{3} \cong \mathbb{Z}$ and a free group of rank 2. More precisely:

With $t=\left(\sigma_{1} \sigma_{2}\right)^{3}$ and $a=\sigma_{1}^{2}, b=\sigma_{2}^{2}$ a presentation for $P_{3}$ is given by

$$
P_{3}=\langle t, a, b \mid t a=a t, t b=b t\rangle .
$$

Thus, by deleting the column corresponding to $t$ from the Fox Jacobian, the reduced matrix $A$ is:

$$
A=\left(\begin{array}{cc}
t-1 & 0 \\
0 & t-1
\end{array}\right)
$$

By Theorem 1.1 the right-hand-side of Lücks formula (4) equals the logarithmic Mahler measure of $\operatorname{det}(A)=(t-1)^{2}$, which is 0 .

Now, since $P_{3}$ has index 6 in $S^{3}-K$, for $K$ the trefoil, we know [Lüc02] that Formula (4) applied to the complement of the trefoil must be $\frac{1}{6}$ of this and thus also 0.

Acknowledgment: The author thanks Pat Gilmer, Walter Neumann, Barbara Niethammer and Neal Stoltzfus for valuable comments and suggestions. Moreover, he is very grateful for the remarks of an anonymous referee. The work on this paper was partially supported by NSF DMS-0306774 and DMS-FRG-0456275. 


\section{References}

[BZ85] Gerhard Burde and Heiner Zieschang, Knots, de Gruyter Studies in Mathematics, vol. 5, Walter de Gruyter \& Co., Berlin, 1985.

[Den06] Christopher Deninger, Fuglede-Kadison determinants and entropy for actions of discrete amenable groups, J. Amer. Math. Soc. 19 (2006), no. 3, 737-758 (electronic), arXiv:math.DS/0502233.

[DL08] Oliver T. Dasbach and Matilde N. Lalin, Mahler measure under variations of the base group, to appear in: Forum Mathematicum (2008), arXiv:math.NT/0702394.

[DM01] Oliver T. Dasbach and Brian S. Mangum, The automorphism group of a free group is not subgroup separable, Knots, braids, and mapping class groups - papers dedicated to Joan S. Birman (New York, 1998), AMS/IP Stud. Adv. Math., vol. 24, Amer. Math. Soc., Providence, RI, 2001, pp. 23-27.

[EW99] Graham Everest and Thomas Ward, Heights of polynomials and entropy in algebraic dynamics, Universitext, Springer-Verlag London Ltd., London, 1999.

[Leh85] D. H. Lehmer, Interesting series involving the central binomial coefficient, Amer. Math. Monthly 92 (1985), no. 7, 449-457.

[Lüc94] Wolfgang Lück, $L^{2}$-torsion and 3-manifolds, Low-dimensional topology (Knoxville, TN, 1992), Conf. Proc. Lecture Notes Geom. Topology, III, Internat. Press, Cambridge, MA, 1994, pp. 75-107.

[Lüc02] — , L L-invariants: Theory and Applications to Geometry and K-theory, Ergebnisse der Mathematik und ihrer Grenzgebiete. 3. Folge. A Series of Modern Surveys in Mathematics [Results in Mathematics and Related Areas. 3rd Series. A Series of Modern Surveys in Mathematics], vol. 44, Springer-Verlag, Berlin, 2002.

[RV99] Fernando Rodriguez Villegas, Modular Mahler measures. I, Topics in number theory (University Park, PA, 1997), Math. Appl., vol. 467, Kluwer Acad. Publ., Dordrecht, 1999, pp. 17-48.

[Wil94] Herbert S. Wilf, generatingfunctionology, second ed., Academic Press Inc., Boston, MA, 1994.

[Wol99] Stephen Wolfram, The Mathematica ${ }^{\circledR}$ book, fourth ed., Wolfram Media, Inc., Champaign, IL, 1999. 